\title{
EFFETS DES EXTRAITS TOTAUX AQUEUX DE Mallotus oppositifolium ET DE Euphorbia hirta (Euphorbiaceae) SUR L'ACTIVITE CONTRACTILE INTESTINALE DU RAT
}

\author{
R. Kamgang, R. Zintchem, T. Dimo et M. Panjo Yewah \\ Laboratoire de Physiologie animale, Faculté des Sciences - Université de Yaoundé I
}

\begin{abstract}
RÉSUMÉ:- Nous avons testé les extraits totaux aqueux de Mallotus oppositifolium (Mo) et de Euphorbia hirta $(E h)$ in vitro sur les contractions de fragments d'iléon provoquées par l'acétylcholine (ACh) ou le chlorure de potassium $(\mathrm{KCl})$, et in vivo, sur l'induction expérimentale des diarrhées par le sulfate de magnésium (SM). In vitro, les extraits de Mo diminuent le tonus des contractions avec effets dose-dépendante. A $1,32 \mathrm{mg} / \mathrm{ml}$, la diminution du tonus des contractions spontanées est de 40 mm et s'accompagne de relâchement total de l'iléon. A cette dose, ces extraits freinent l'augmentation du tonus des contractions musculaires par l'ACh $\left(6,88.10^{-5} \mathrm{mg} / \mathrm{ml}\right)$ ou le $\mathrm{KCl}(10,31 \mathrm{mM})$, respectivement de $9 \mathrm{~mm}$ et $7 \mathrm{~mm}$. In vivo, à la dose de $712 \mathrm{mg} / \mathrm{kg}$ de poids de l'animal, les extraits de Mo diminuent respectivement de $11 \mathrm{~g}(\mathrm{p}<1 \%)$ et $15 \%(\mathrm{p}<5 \%)$ le poids et le taux des fèces molles des diarrhées provoquées par le SM (6 g/kg de poids). Les extraits totaux aqueux de Eh, in vitro, ont des effets stimulateurs dose-dépendante sur le tonus des contractions spontanées. Avec 3,08 mg/ml de cet extrait, le tonus des contractions spontanées augmentent de $70 \mathrm{~mm}$. Par ailleurs, cet extrait de Eh a un effet activateur sur l'augmentation, par l'ACh $\left(6,8810^{-5} \mathrm{mg} / \mathrm{ml}\right)$ ou le $\mathrm{KCl}(10,31 \mathrm{mM}), \mathrm{du}$ tonus des contractions musculaires : $80 \mathrm{~mm}$ dans chaque cas contre respectivement $32,3 \pm 1,5 \mathrm{~mm}$ et $16,7 \pm 1,5 \mathrm{~mm}$ pour ACh et $\mathrm{KCl}$ seuls. Par contre in vivo, l'extrait de Eh à la dose de $512 \mathrm{mg} / \mathrm{kg}$ de poids de l'animal, diminue le poids et le taux des fèces diarrhéiques respectivement de $12 \mathrm{~g}(\mathrm{p}<1 \%)$ et $30 \%(P<1 \%)$. Ces résultats suggèrent que, chez le rat, sur l'activité contractile de l'intestin, les extraits totaux aqueux de Mallotus oppositifolium auraient des effets antispasmodiques, alors que ceux de Euphorbia hirta auraient des effets spasmogéniques in vitro, et antidiarrhéiques in vivo.
\end{abstract}

Mots clés : Mallotus oppositifolium, Euphorbia hirta, effet antispasmodique, diarrhée, rat.

\begin{abstract}
Effects of total aqueous extracts of Mallotus oppositifolium and Euphorbia hirta (Euphorbiaceae) on intestinal contractile activity of the rat. - The contractile activity of the total aqueous extract of Mallotus oppositifolium (Mo) and Euphorbia hirta (Eh) leaves was investigated. Mo extracts $(1.32 \mathrm{mg} / \mathrm{mL})$ demonstrated inhibiting effect on the stimulation of rat ileal contractions by acetylcholine $(-9 \mathrm{~mm})$ and potassium chloride $(-7 \mathrm{~mm})$. Administrated before the magnesium sulfate $(6 \mathrm{~g} / \mathrm{kg})$, Mo extracts reduce the faeces quantity $(-11 \mathrm{~g}, \mathrm{p}<1 \%)$. Eh activated the stimulation of rat ileal contractions by acetylcholine $(+148 \%)$ and potassium chloride $(+381 \%)$. Administrated before the magnesium sulfate, the Eh aqueous extract reduced the quantity of faeces $(-12 \mathrm{~g}, \mathrm{p}<5 \%)$. The results suggest that on the contractile activity of the rat intestine, total aqueous extracts of Mallotus oppositifolium could have antispasmodic effect, while Euphorbia hirta extracts could have spasmogenic effect in vitro and antidiarrhoeic effects in vivo
\end{abstract}

Key words : Mallotus oppositifolium, Euphorbia hirta, antispasmodic effect, diarrhoea, rat.

\section{INTRODUCTION}

D'après Farnsworth et Kass (1986) $80 \%$ des habitants de la planète auraient recours aux plantes médicinales pour divers problèmes de santé. Parmi les plantes couramment utilisées dans le traitement des diarrhées Mallotus oppositifolium (Mo) et Euphorbia hirta (Eh) ont retenu notre attention. La première (Mo) est utilisée pour le traitement de divers maux (Mshana et al., 2000) : les feuilles sont recommandées pour les migraines, les blessures, les hémorragies ; les feuilles et autres parties aériennes pour les helminthiases ; les racines pour le lumbago et la plante 
Effets des Extraits Totaux Aqueux de Mallotus Oppositifolium et de

Euphorbia lirta (euphorbiaceae) sur L'activite Contractile Intestinale du Rat

entière pour la dysenterie. Traditionnellement et populairement Mo est utilisée au Cameroun dans les régions de savane et forêt pour le traitement des diarrhées infantiles. Pour la deuxième (Eh), la plante entière est utilisée en Afrique et en Asie dans le traitement des désordres gastro-intestinaux (obstruction, amibiase, diarrhées, vomissements, constipation), les ulcères buccaux, les MST, l'impuissance sexuelle chez l'homme, les blessures, l'hypolactation, les menaces d'avortement, l'énurésie (Adjanohoun et al., 1996 ; Dalziel, 1937), les maladies respiratoires, et les conjonctivites (Galvez et al., 1993a). Il lui est reconnu aussi (Galvez et al.,1993b) des propriétés hypotensives, antipyrétiques, anti-inflammatoires, hypoglycémiantes et antibactériennes ; ces auteurs en ont extrait un flavonoïde à effet inhibiteur sur les diarrhées induites par l'huile de castor et le prostaglandine E2.

Dans ce travail, nous avons examiné les effets des extraits totaux aqueux de Mallotus oppositifolium et de Euphorbia hirta chez les rats, d'abord in vitro, sur l'activation des contractions des fragments d'iléon, puis in vivo, sur l'induction des diarrhées par le sulfate de magnésium.

\section{MATÉRIELETMÉTHODE}

\section{Les extraits}

Les feuilles de Mallotus oppositifolium (Mo) et de Euphorbia hirta (Eh) sont récoltées aux environs de Yaoundé. Inspiré de la méthode traditionnelle, $50 \mathrm{~g}$ de ces feuilles sont pilées et macérées dans $200 \mathrm{~mL}$ d'eau distillée. Chaque macérât est conservé à $4^{\circ} \mathrm{C}$ pendant $12 \mathrm{~h}$, puis porté à $20^{\circ} \mathrm{C}$ dans l'étuve pendant une semaine. Nous avons obtenu respectivement $17,8 \mathrm{mg}$ et $12,8 \mathrm{mg}$ de matière sèche par ml d'extrait total aqueux de Mo et Eh.

\section{Essais pharmacologiques}

\section{In vitro}

2 à 3 fragments d'iléon de $1,5 \mathrm{~cm}$ chacun prélevés chez des rats wistar (150 - $200 \mathrm{~g})$ sont conservés pendant les essais dans le liquide physiologique de Tyrode $(8 \mathrm{~g} \mathrm{NaCl} ; 0,2 \mathrm{~g}$ $\mathrm{KCl} ; 0,1 \mathrm{~g} \mathrm{MgSO}_{4} ; 0,05 \mathrm{~g} \mathrm{NaH}_{2} \mathrm{PO}_{4} ; 1 \mathrm{~g} \mathrm{NaHCO}_{3} ; 0,2$ $\mathrm{gCaCl}_{2} .1 \mathrm{~g}$ glucose ; E.D. qsp $1000 \mathrm{~mL}$ ) oxygéné et maintenu à $37^{\circ} \mathrm{C}$. Par unité, ces fragments d'iléon sont montés dans une cuve à organe isolé et relié à un transducteur isotonique (type Ugo Basile 7006). Les contractions toniques sont enregistrées sur dynamographe (Ugo Basile type Gemini 7070 multivitesse à 2 pistes). Un volume de chaque extrait est ajouté au milieu de perfusion et les effets sont observés. Après 2 à 3 lavages au Tyrode et stabilisation pendant $20 \mathrm{~min}$, une dose supérieure d'extrait est ajoutée dans la chambre à organe. Les effets de différentes doses des extraits totaux aqueux des feuilles de Mo $(0,18 \mathrm{mg} / \mathrm{mL} ; 0,35 \mathrm{mg} / \mathrm{mL} ; 0,68 \mathrm{mg} / \mathrm{mL}$ $; 1,32 \mathrm{mg} / \mathrm{mL} ; 2,46 \mathrm{mg} / \mathrm{mL})$, des feuilles et tiges de $\mathrm{Eh}(0,25$ $\mathrm{mg} / \mathrm{mL} ; 0,49 \mathrm{mg} / \mathrm{mL} ; 0,94 \mathrm{mg} / \mathrm{mL} ; 1,75 \mathrm{mg} / \mathrm{mL} ; 3,08 \mathrm{mg} /$ $\mathrm{mL}$ ) sont évaluées sur l'activité contracturante, sur le muscle lisse iléal, de l'acétylcholine (ACh) et du chlorure de potassium $(\mathrm{KCl})$.

In vivo

Les rats sont placés individuellement dans 5 cages métaboliques pendant les expérimentations. Les animaux sont nourris et abreuvés ad libitum. Une étoffe blanche est placée sous la grille de support des animaux de manière à retenir les fèces en laissant passer les urines qui sont recueillies dans un flacon. Après observation de son aspect, les matières fécales sont pesées. Le sulfate de magnésium (SM), agent cathartique, est administré per os à la dose de $6 \mathrm{~g} . \mathrm{kg}^{-1}$ de poids de l' animal pour provoquer la diarrhée. Pour l'examen de l'effet anti-cathartique, les extraits de Mo et de Eh sont administrés per os aux doses respectives de 712 et $512 \mathrm{mg} \cdot \mathrm{kg}^{-1} 60 \mathrm{~min}$ avant le SM.

\section{Traitement statistique des résultats}

Les résultats sont les moyennes arithmétiques (m) des valeurs individuelles affectées de l'erreur standard sur la moyenne $(\mathrm{m} \pm \mathrm{ESM})$. Pour les travaux in vivo les résultats sont analysés par le test non paramétrique U de Mann et Whitney.

\section{RÉSULTATS}

En présence d'extraits totaux aqueux de Mo le tonus des contractions spontanées des fragments d'iléon in vitro diminue. Cette diminution est dose dépendante et atteint un plancher à $40 \mathrm{~mm}$ avec un relâchement total du muscle à $1,32 \mathrm{mg} / \mathrm{ml}$ d'extrait (fig. 1). Avec les extraits totaux aqueux de Eh on observe une augmentation dose dépendante du tonus et de l'amplitude de contraction. A 3,08 mg/ml d'extrait, le tonus atteint un plateau à 70 mm (fig. 1).

Lorsque le $\mathrm{ACh}\left(6,88 \times 10^{-5} \mathrm{mg} / \mathrm{ml}\right)$ et le $\mathrm{KCl}(10,31 \mathrm{mM})$ sont administrés dans le milieu après l'extrait total aqueux de Mo (1,32 mg/ml), l'augmentation du tonus de contraction est réduite respectivement de $9 \mathrm{~mm}$ et $7 \mathrm{~mm}$ (tableau 1$)$. Si le $\mathrm{ACh}$ et le $\mathrm{KCl}$ sont administrés après $\mathrm{Eh}(0,94 \mathrm{mg} / \mathrm{ml})$ les tonus de contraction augmentent dans chaque cas de 80 $\mathrm{mm}$; soit des hausses respective de $+148 \%$ et $+381 \%$ par rapport aux effets de $\mathrm{ACh}$ et $\mathrm{KCl}$. 


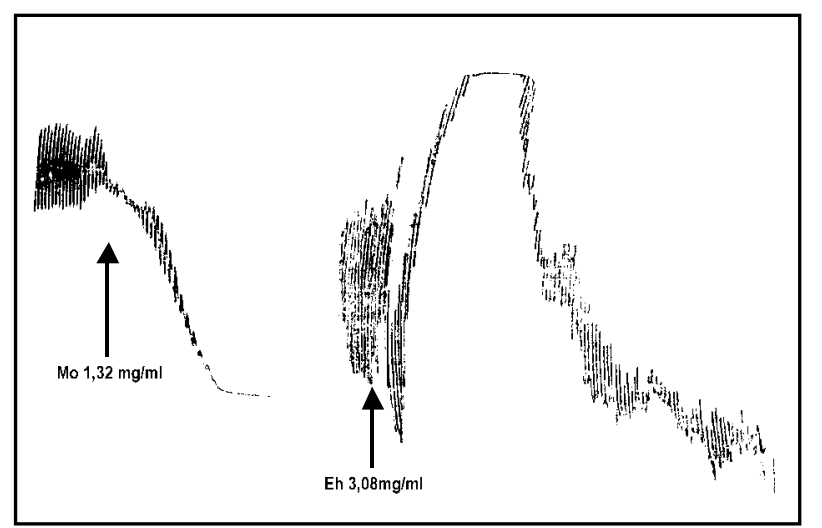

Figure 1 : Effets de Mallotus oppositifolium (Mo) et de Euphorbia hirta (Eh) sur les contractions spontanées de fragments d'iléon de rat in vitro.

Tableau 1 : Effets de Mallotus oppositifolium (Mo : 1,32 mg/mL) et de Euphorbia hirta (Eh : 0,94 mg/mL) sur la stimulation in vitro des contractions des fragments d'iléon de rat par l'acétylcholine (ACh : $\left.6,88 \times 10^{-5} \mathrm{mg} / \mathrm{mL}\right)$ et le chlorure de potassium $(\mathrm{KCl}: 10,31 \mathrm{mM})$

\begin{tabular}{lccc}
\hline Traitement & $\mathrm{n}$ & tonus $(\mathrm{mm})$ & Variation \\
\hline $\mathrm{ACh}$ & 3 & $32,3 \pm 1,5$ & \\
$\mathrm{KCl}$ & 3 & $16,7 \pm 1,5$ & \\
\hline $\mathrm{Mo}+\mathrm{Ach}$ & 3 & $23,3 \pm 1,2$ & $-9 \mathrm{~mm} / \mathrm{ACh}$ \\
$\mathrm{Mo}+\mathrm{KCl}$ & 3 & $9,7 \pm 0,9$ & $-7 \mathrm{~mm} / \mathrm{KCl}$ \\
\hline $\mathrm{Eh}+\mathrm{Ach}$ & 2 & 80 & $+47,7 \mathrm{~mm} / \mathrm{ACh}$ \\
$\mathrm{Eh}+\mathrm{KCl}$ & 2 & 80 & $+63,7 \mathrm{~mm} / \mathrm{KCl}$ \\
\hline
\end{tabular}

In vivo, après plusieurs dose successives, c'est à $6 \mathrm{~g}$ de SM par kg de poids de l'animal que nous avons obtenu, après $3 \mathrm{~h}$, des diarrhées chez nos rats. Ces diarrhées se manifestent par l'augmentation de fèces émises en quantité $(23,8 \mathrm{~g} \pm 2,8$ contre $4,5 \pm 0,6 ; \mathrm{p}<1 \%)$ et en qualité : $72 \pm 5$ $\%$ de fèces molles. Lorsque le SM est administré $1 \mathrm{~h}$ après les extraits totaux aqueux de Mo $(712 \mathrm{mg} / \mathrm{kg})$, les diarrhées apparaissent après plus de $4 \mathrm{~h}$ (tableau 2$)$, avec réduction des fèces par rapport à l'agent cathartique aussi bien en quantité $(18,8 \pm 1,5 \mathrm{~g}$ contre $23,8 \pm 2,8 \mathrm{~g} ; \mathrm{p}<1 \%)$ qu'en qualité : $57 \%$ de fèces molles $(\mathrm{p}<5 \%)$.

Le SM administré après les extraits totaux aqueux de Eh $(512 \mathrm{mg} / \mathrm{kg})$ raccourcissent le temps d'apparition des diarrhées : $195 \pm 7$ contre $204 \pm 12 \min (\mathrm{p}<1 \%)$, respectivement chez l'animal traité à l'extrait et SM et au SM seul. On note la réduction de la quantité et de la qualité de matière fécale : $11,7 \pm 1,9 \mathrm{~g}$ de fèces avec $42 \%$ de fèces molles $(\mathrm{p}<5 \%)$.
Tableau 2 : Effets de Mallotus oppositifolium (Mo: $712 \mathrm{mg} / \mathrm{kg}$ ) et Euphorbia hirta (Eh : $512 \mathrm{mg} / \mathrm{kg}$ ) sur l'induction de diarrhée par le sulfate de magnésium (SM : $6 \mathrm{~g} / \mathrm{kg})$ chez le rat $(\mathrm{n}=10)$. Différence significative : $* \mathrm{p}<5 \%$ par rapport à $\mathrm{SM} ; * * \mathrm{p}<1 \%$, a par rapport au témoin, b par rapport à SM.

\begin{tabular}{lccc}
\hline Traitement & $\begin{array}{c}\text { Poids total des } \\
\text { fèces }(\mathrm{g})\end{array}$ & $\begin{array}{c}\text { Taux de fèces } \\
\text { molles }(\%)\end{array}$ & $\begin{array}{c}\text { Début } \\
\text { d'émission }(\mathrm{min})\end{array}$ \\
\hline Témoins & $4,5 \pm 0,6$ & & \\
$\mathrm{SM}$ & $23,8 \pm 2,8 * *^{\mathrm{a}}$ & $71,9 \pm 4,6$ & $204 \pm 12$ \\
Mo + SM & $12,9 \pm 1,5^{*} *^{\mathrm{b}}$ & $57 \pm 5^{*}$ & $283 \pm 8 * *^{\mathrm{b}}$ \\
Eh + SM & $11,7 \pm 1,9 *$ & $42 \pm 4 * *^{\mathrm{b}}$ & $195 \pm 7 * *^{\mathrm{b}}$ \\
\hline
\end{tabular}

\section{DISCUSSION}

A $1,32 \mathrm{mg} / \mathrm{ml}$ les extraits totaux aqueux de Mallotus oppositifolium (Mo) inhibent totalement in vitro les contractions iléales et réduisent l'augmentation du tonus de contraction de ce muscle par l'acétylcholine (ACh : 6,88 $10^{-5} \mathrm{mg} / \mathrm{ml}$ ) et le chlorure de potassium $(\mathrm{KCl}: 10,31 \mathrm{mM})$ qui sont des substances contracturantes potentiels sensibles souvent à l'origine des diarrhées (Devor et al., 1992 ; Schmitt, 1978). En réduisant in vivo chez le rat la quantité de fèces émises et en rallongeant en même temps le temps d'apparition des diarrhées, les extraits aqueux de Mo auraient ainsi une action inhibitrice sur l'induction des diarrhées par le sulfate de magnésium (SM : $6 \mathrm{~g} / \mathrm{kg}$ ). Ce freinage in vitro de l'augmentation par l' $\mathrm{ACh}$ et le $\mathrm{KCl} \mathrm{du}$ tonus de contraction iléale et la modération in vivo de l'induction par le SM des diarrhées traduite par l'émission fécale, suggèrent que les extraits totaux aqueux de Mo auraient chez le rat des effets antispasmodiques sur l'activité contractile de l'intestin.

Les extraits de Euphorbia hirta (Eh) ont in vitro des effets à tendance stimulatrice sur les contractions iléales puisqu'ils provoquent l'augmentation du tonus de contraction, suivie par la suite du relâchement du muscle. On observe aussi, in vitro, que ces extraits de Eh accroît l'action stimulatrice de $\mathrm{ACh}$ et de $\mathrm{KCl}$ sur les contractions des fragments d'iléon. In vivo, les extraits totaux aqueux de Eh auraient un rôle permissif sur l'action du SM puisqu'ils raccourcissent le temps d'effet du SM sur l'induction des diarrhées,. Par ailleurs, in vivo, ces extraits réduisent significativement l'induction de diarrhées par le SM tant en quantité $(\mathrm{p}<5 \%)$ qu'en qualité $(\mathrm{p}<1 \%)$ de fèces et auraient ainsi chez le rat des effets anti-diarrhéiques observés par Tona et al. (1999). Les extraits totaux aqueux de Eh auraient des effets spasmogéniques in vitro, et antidiarrhéiques in vivo. 
Effets des Extraits Totaux Aqueux de Mallotus Oppositifolium et de Euphorbia lirta (euphorbiaceae) sur L'activite Contractile Intestinale du Rat

\section{RÉFÉRENCES}

ADJANOHOUN JE, ABOUBAKA N, DRAMANE K, EBOT NE, EKPERE JA, ENOW-OROCK EG, FOCHO D, GBILEZO, KAMANYI A, KAMSU-KOM J, KEITA A, MBENKUM T, MBI CN, MBIELE AL, MBOME LL, MUBIRU NK, NANCY WL, NKONGMENECK B, SATABIE B, SOFOWORA A, TAMZE V, WIRMUM CK (1996) Traditional Medicine and Pharmacopoeia : Contribution to Ethnobotanical and Floristic studies in Cameroon. Organization of African Unity Scientific, Technical and Research Commission. Centre National de Production de Manuel Scolaires, Porto-Novo, p. 179.

DALZIEL JM (1937) The useful plants of West Tropical Africa. Appendix to the flora of West Tropical Africa. Crown agents for Overseas Governments and Administrations, London, UK, p. 143.

DEVOR CD and DUFFEY ME (1992) Carbochol induces $\mathrm{K}^{+}, \mathrm{Cl}^{-}$and non-selective cations conductance in $\mathrm{T} 84$ cells : a perforated patch-clamp study. American Journal of Physiology, 263 (Cell Physiol. 32), C780C787.

FARNSWORTH NR and KASS CJ (1986) An approach utilizing information from traditional medicine to identify tumor inhibiting plants. Bulletin. de l'OMS, 66, 159.

GALVEZ J, ZARZUELO A, CRESPO ME, LORENTE MD, OCETE MA, JIMENEZ J (1993a) Antidiarrhoeic activity of Euphorbia hirta extract and isolation of an active flavonoid constituent. Planta Medica, 59, 333-336.

GALVEZ J, CRESPO ME, JIMENEZ J, SUAREZ A, ZARZUELO A (1993b) Antidiarrhoeic activity of quercitrin in mice and. Journal of Pharmacy and Pharmacology. 45, 157-159.

MSHANA NR, ABBIW DK, ADDAE-MENSAH I, ADJANOHOUN JE, AHYI MRA, EKPERE JA, ENOW-OROCKEG, GBILEZO, NOAMESIGK, ODEI MA, ODUNLAMI H, OTENG-YEBOAH AA, SARPONG K, SAFOWORA A and TAEKIE AN (2000) Traditional Medicine and Pharmacopoeia : Contribution to the revision of Ethnobotanical and floristic studies in Ghana. Organization of African Unity/Scientific, Technical and Research Commission. P. 243.

SCHMITT H (1978) les récepteurs muscariniques. Actualité pharmacologique, 30 ${ }^{\mathrm{e}}$ Série, 113-158.
TONA L, KAMBU K, MESIA K, CINANGA K, APERS S, DE BRUYNE T, PIETERS L, TOTTE $J$ and VLIETINCK AJ (1999) Biological screening of traditional preparations from some medicinal plants used as antidiarrhoeal in Kinshasa, Congo. Phytomedicine, 6(1), 59-66. 